\title{
Aplicación de las tecnologías en la educación en odontología durante la pandemia por COVID-19.
}

\section{Application of technologies in dental education during the COVID-19 pandemic.}

\author{
Agustín Tiol-Carrillo*
}

\section{RESUMEN}

Las tecnologías en la educación son un conjunto de recursos, procesos y herramientas de la información y comunicación aplicadas a las actividades del sistema educativo para favorecer el proceso de enseñanzaaprendizaje. Las técnicas de enseñanza-aprendizaje convencionales sufrieron un cambio drástico tras la pandemia por COVID-19 y la educación odontológica se ha visto seriamente afectada. Si bien la práctica clínica de los estudiantes de odontología no puede ser sustituida por ningún motivo, existen múltiples herramientas, aplicaciones y plataformas digitales que permiten a los profesores impartir y diseñar cursos a distancia para favorecer el aprendizaje y la interiorización de conocimiento por parte de sus alumnos. El objetivo del presente artículo es explicar las diferentes tecnologías educativas existentes y la forma en cómo estas pueden ser aplicadas en el proceso de enseñanzaaprendizaje en odontología.

Palabras clave: Educación, tecnologías en educación, pedagogía en odontología.

\section{ABSTRACT}

Technology in education is a set of information and communication resources, processes and tools applied to the activities of the educational system to favor the teaching-learning process. Conventional learning teaching techniques underwent a drastic change after the COVID-19 pandemic, and dental education has been significantly affected. Although the clinical practice of dental students cannot be replaced for any reason, there are multiple tools, applications and digital platforms that allow teachers to teach and design distance courses to promote learning and internalization of knowledge by students. The aim of this article is to explain the different existing educational technologies and how these can be applied in the teaching-learning process in dentistry.

Keywords: Education, technologies in education, pedagogy in dentistry.

\section{INTRODUCCIÓN}

$\mathrm{L}$ a pandemia por COVID-19 produjo cambios drásticos en el estilo de vida de todos los seres humanos. Con el fin de disminuir contagios se tomaron medidas para restringir el contacto directo entre personas en lugares públicos, oficinas e instituciones de enseñanza, lo cual ha mermado de manera significativa la educación a todos los niveles.

De acuerdo con el Programa Nacional de Educación, la educación superior proporciona a los mexicanos los elementos para su desarrollo integral, y a su vez forma científicos, humanistas y profesionales en múltiples áreas, dotados de conocimientos de vanguardia y dispuestos a solucionar las necesidades del país, ${ }^{1}$ por tanto, resulta fácil comprender los efectos negativos que las restricciones impuestas por el gobierno federal produjeron en la educación universitaria durante el año 2020 y parte del 2021.

La licenciatura en odontología, en cualquier institución pública o privada, pretende dotar al alumno de los

\footnotetext{
* Especialista en Estomatología Pediátrica, Instituto Nacional de Pediatría. Profesor de licenciatura en UAM Xochimilco, Universidad Anáhuac Norte y Universidad Intercontinental.

Recibido: 17 de febrero de 2021. Aceptado: 06 de abril de 2021.

Citar como: Tiol-Carrillo A. Aplicación de las tecnologías en la educación en odontología durante la pandemia por COVID-19. Rev ADM. 2021; 78 (3): 155-161. https://dx.doi.org/10.35366/100073
} 
conocimientos, habilidades, destrezas y aptitudes necesarias para ejercer la profesión, ${ }^{2}$ y resultan alarmantes para los catedráticos y alumnos inscritos a dicha licenciatura, las condiciones en las cuales se han impartido las asignaturas de forma virtual.

Nuestra profesión requiere forzosamente de una destreza manual que bajo ninguna circunstancia puede ser sustituida por métodos educativos a distancia, ya que la odontología exige el desarrollo y entrenamiento en las habilidades técnicas y clínicas, donde la participación de los pacientes que demandan una solución satisfactoria a su padecimiento es de vital importancia., 2,3

Sin embargo, a pesar de los múltiples aspectos negativos que la pandemia por COVID-19 ha generado en la educación, es un buen pretexto para que todos aquellos odontólogos que nos dedicamos a formar nuevos profesionistas nos cuestionemos sobre las técnicas de enseñanza-aprendizaje utilizadas antes de la pandemia y las adecuaciones educacionales que tuvieron lugar a raíz de ésta. Resulta interesante darse cuenta de que un gran número de docentes universitarios, adscritos a las licenciaturas en odontología, tuvieron grandes complicaciones y tropiezos al cambiar súbitamente sus métodos de enseñanza mediante el uso de las tecnologías en la educación para impartir sus clases.

El objetivo del presente artículo es analizar los conceptos relacionados con las tecnologías en la educación, y dilucidar cómo éstas pueden utilizarse en la formación universitaria de odontólogos que si bien jamás podrán sustituir la actividad clínica, pueden reforzar de forma relevante las técnicas de enseñanza-aprendizaje en las futuras generaciones, dando lugar a una importante revolución en la educación odontológica que sea capaz de incorporar la tecnología a las técnicas pedagógicas convencionales.

\section{LAS TECNOLOGÍAS DE LA EDUCACIÓN}

Hoy en día cualquier actividad de la vida cotidiana está relacionada directa o indirectamente con la tecnología, donde el uso de Internet, computadoras, teléfonos celulares, tabletas y cualquier otro dispositivo electrónico resultan imprescindibles. Asimismo, estos dispositivos tecnológicos permiten la comunicación entre las personas (a través de llamadas, videollamadas o mensajes de texto), además de facilitar el acceso a un sinfín de información a través de Internet. Por lo anterior, al conjunto de alternativas y métodos de búsqueda de información que facilitan la comunicación interpersonal se les conoce como Tecnologías de la Información y la Comunicación (TIC).

La tecnología educativa es una disciplina pedagógica novedosa que permite llevar a cabo el proceso de enseñanza-aprendizaje valiéndose de los medios tecnológicos para lograr los objetivos educativos, ${ }^{4}$ y en pleno siglo XXI todas las instituciones de enseñanza deben implementarlos para evitar quedar rezagados en el camino irrefrenable de la tecnología.

La generación de alumnos actualmente inscritos en la educación básica, media superior y superior tiene al alcance un sinfín de información en un sinnúmero de páginas de Internet, y son plenamente capaces de aprovechar esta información para crear nuevos conocimientos, por lo que a esta generación se le conoce como sociedad del conocimiento, que se caracteriza por una capacidad competitiva no sólo de adquirir conocimientos, sino también de transmitirlos y aplicarlos ${ }^{5}$ y por tanto, todo profesor universitario debe estar lo suficientemente preparado y actualizado en los temas de la materia que imparte debido a que sus alumnos, generalmente expertos en la nueva era digital, pueden fácilmente debatir y discutir los conceptos mencionados en clase.

\section{Asignaturas en línea}

Anteriormente se consideraba al salón de clases como el único sitio donde era posible impartir una cátedra; sin embargo, hoy en día nos hemos dado cuenta de que es completamente viable el uso de la tecnología para dicho fin.

En muchas universidades del mundo se imparten asignaturas en diferentes modalidades, pudiendo ser totalmente presenciales, totalmente en línea y de forma híbrida (presencial y en línea). La modalidad en línea o la forma híbrida ofrece varias ventajas como facilitar la comunicación entre el profesor y los alumnos, aportar flexibilidad en tiempos y horarios, brindar asistencia a cada alumno de manera individualizada, delegar a los sistemas tecnológicos la entrega oportuna de tareas, fomentando la responsabilidad de los alumnos en la entrega de sus actividades, y desde luego mejorar permanentemente las competencias digitales de alumnos y catedráticos. ${ }^{6}$

Existen múltiples plataformas virtuales para la impartición de materias en línea, donde se encuentran disponibles numerosas herramientas agrupadas y optimizadas que permiten al docente la creación y gestión de cursos sin que sean necesarios conocimientos especializados de programación $^{7}$ (Tabla 1). 


\section{Tabla 1: Características de las plataformas educativas para impartición de clases y cursos en línea.}

\begin{tabular}{ll} 
Herramienta digital & Utilización \\
\hline De gestión de contenidos & Ofrecer al alumno archivos en diferentes formatos (HTML, PDF, TXT, \\
& ODT, PNG, etc.) organizándolos en directorios y carpetas \\
De comunicación y colaboración & $\begin{array}{l}\text { Aperturar foros de debate e intercambio de información, salas de chat, } \\
\text { mensajería grupal o individual entre el profesor y sus alumnos }\end{array}$ \\
De seguimiento y evaluación & $\begin{array}{l}\text { Creación de cuestionarios y exámenes para evaluar a los alumnos, asignación y entrega de } \\
\text { tareas. Obtención de un récord individual de cada alumno que permita visualizar su desempeño }\end{array}$ \\
De herramientas de administración & $\begin{array}{l}\text { Permite controlar mediante autentificación el nombre de usuario } \\
\text { y asignación de permisos }\end{array}$ \\
Complementarias & y contraseña de los alumnos inscritos en el curso \\
& Servicios adicionales como portafolios, bloc de notas, búsqueda de contenidos
\end{tabular}

Son múltiples las plataformas virtuales de enseñanza disponibles actualmente, y su selección es una decisión de la institución universitaria más que del profesorado; sin embargo, la institución está obligada a capacitar a sus profesores en el uso de las mismas para poder explotar al máximo sus herramientas. Ejemplos de plataformas utilizadas por su fácil manejo y suficiente contenido son la plataforma@prende 2.0 (a través de la cual la Secretaría de Educación Pública busca fomentar el uso de las TIC), D2L Brightspace, Microsoft Teams, Google Classroom, Canvas, entre otros.

\section{CÁTEDRAS VIRTUALES EN TIEMPO REAL}

Se trata del método más utilizado para la impartición de clases a distancia durante la pandemia por COVID-19 y es posible gracias a diversos programas que permiten a los miembros de un grupo conectarse a la reunión virtual a través de cualquier dispositivo digital como computadoras portátiles o de escritorio, tabletas y teléfonos celulares. Estos programas o aplicaciones de videoconferencia permiten la interacción de todos los participantes que intervengan en ella de manera ordenada, pues tiene funciones como «levantar la mano» para pedir la palabra, gestionar grupos, implementar encuestas, compartir contenido audiovisual en tiempo real, hacer uso de una pizarra digital, uso del chat público y privado y grabar las sesiones virtuales total o parcialmente. Son múltiples los programas y aplicaciones utilizadas para este fin, dentro de las cuales destacan Zoom Video y la mayoría de las plataformas educativas anteriormente mencionadas cuentan con herramientas para videoconferencia.

Es importante tener en cuenta algunas recomendaciones con fines pedagógicos en el uso de esta modalidad como permitir a los alumnos visualizar al profesor durante toda la sesión, el cual deberá estar en todo momento presentable, lo cual incitará a los alumnos a presentarse a clase en las mismas condiciones. Hacer partícipes a los alumnos en todo momento durante las sesiones virtuales a través de preguntas o comentarios, ya que su atención puede verse mermada de manera significativa si no se les involucra en la dinámica de clase, así como otorgar algunos minutos de descanso a los participantes de la videoconferencia. Asimismo, el profesor debe impartir su cátedra en un sitio silencioso con suficiente iluminación y evitar en todo momento la presencia de distractores que puedan desviar la atención de los estudiantes como consumir alimentos mientras se imparte la clase o la presencia de numerosos objetos, personas o imágenes a espaldas del profesor, por mencionar algunos.

\section{GAMIFICACIÓN DE LAS SESIONES VIRTUALES}

Todo profesor, a la hora de impartir una clase virtual, debe ser consciente de los grandes obstáculos que enfrenta para que sus alumnos presten atención en todo momento, ya que existen en casa un sinnúmero de distractores que sin duda desvían su atención.

No existe una acción más antipedagógica que impartir la cátedra sin considerar la participación de los alumnos, tomando en cuenta además que se trata de individuos jóvenes, en quienes es más fácil atraer su atención mediante el uso de dinámicas lúdicas que favorezcan su atención y aprendizaje, esto se logra a través de la gamificación, una disciplina pedagógica cuyo objetivo es favorecer el aprendizaje mediante el uso de elementos lúdicos y juegos, cuya ventaja 
principal es la motivación de los alumnos durante su implementación. ${ }^{8}$

El académico debe explotar su creatividad al máximo para implementar dinámicas lúdicas que favorezcan la interiorización de conocimientos en sus alumnos, ejemplos de esto son juegos, concursos, cuestionarios, misiones o retos que, tras su resolución, se obtenga alguna recompensa, punto, regalo, entre otros. Ejemplos de éstos son concursos virtuales a través de Kahoot, plataforma que permite crear concursos de conocimientos entre alumnos de forma individual o en equipos, a través de preguntas con respuestas de opción múltiple y verdadero o falso elaboradas por el profesor, Socrative que permite responder cuestionarios que refuercen conceptos vistos en clase, la creación de concursos de conocimientos generales de la materia a través de la simulación de un programa de preguntas como «Jeopardy» mediante el uso de Power Point (Figura 1).

\section{APLICACIONES INTERACTIVAS PARA CLASES VIRTUALES}

Muchos profesores, independientemente de la materia que imparten, cuentan con las mismas dinámicas y presentaciones ya establecidas, incluso por años, para impartir sus clases; sin embargo, siempre es importante salir de la zona de confort y periódicamente revisar, modificar y reestructurar las presentaciones y dinámicas de clases para actualizar su contenido, este proceso de constante cambio y reestructuración del material y dinámicas de clase es lo que en pedagogía se conoce como reingeniería docente.

Un error frecuente es que el docente considera haber alcanzado el dominio completo de su asignatura y por tanto, rara vez se mantiene en constante actualización de los temas enfocándose exclusivamente en repetir las mismas actividades una y otra vez. ${ }^{9}$

Es considerado el padre de la odontología
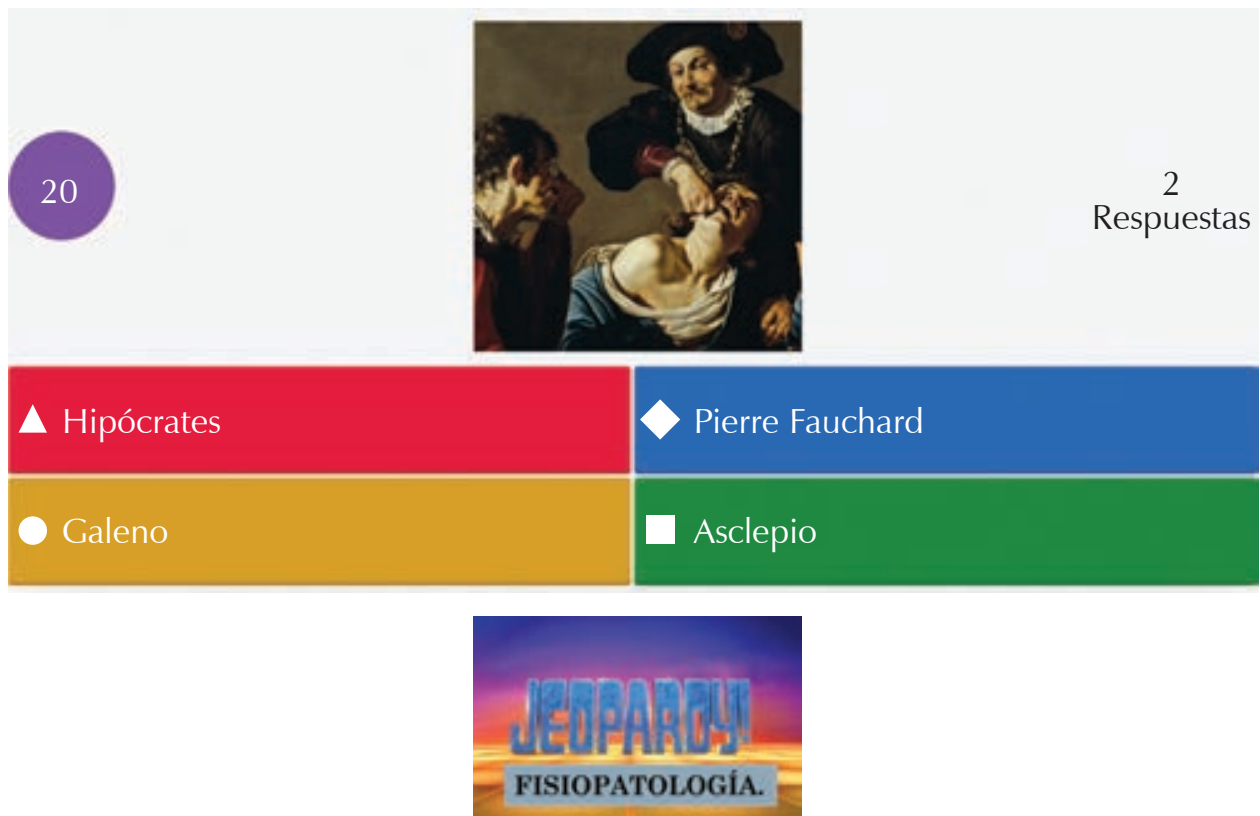

\begin{tabular}{cccccc}
$\begin{array}{c}\text { Enfermedades } \\
\text { hereditarias }\end{array}$ & $\begin{array}{c}\text { Enfermedades } \\
\text { infecciosas }\end{array}$ & $\begin{array}{c}\text { Proceso } \\
\text { patológico }\end{array}$ & $\begin{array}{c}\text { Muerte } \\
\text { celular }\end{array}$ & $\begin{array}{c}\text { Diagnóstico de } \\
\text { enfermedades }\end{array}$ & $\begin{array}{c}\text { Cultura } \\
\text { general }\end{array}$ \\
\hline 100 & 100 & 100 & 100 & 100 & 100 \\
200 & 200 & 200 & 200 & 200 & 200 \\
300 & 300 & 300 & 300 & 300 & 300 \\
400 & 400 & 400 & 400 & 400 & 400 \\
500 & 500 & 500 & 500 & 500 & 500 \\
\hline
\end{tabular}

Figura 1:

Creación de actividades lúdicas a modo de juegos o concursos a través de Kahoot y Power Point. 


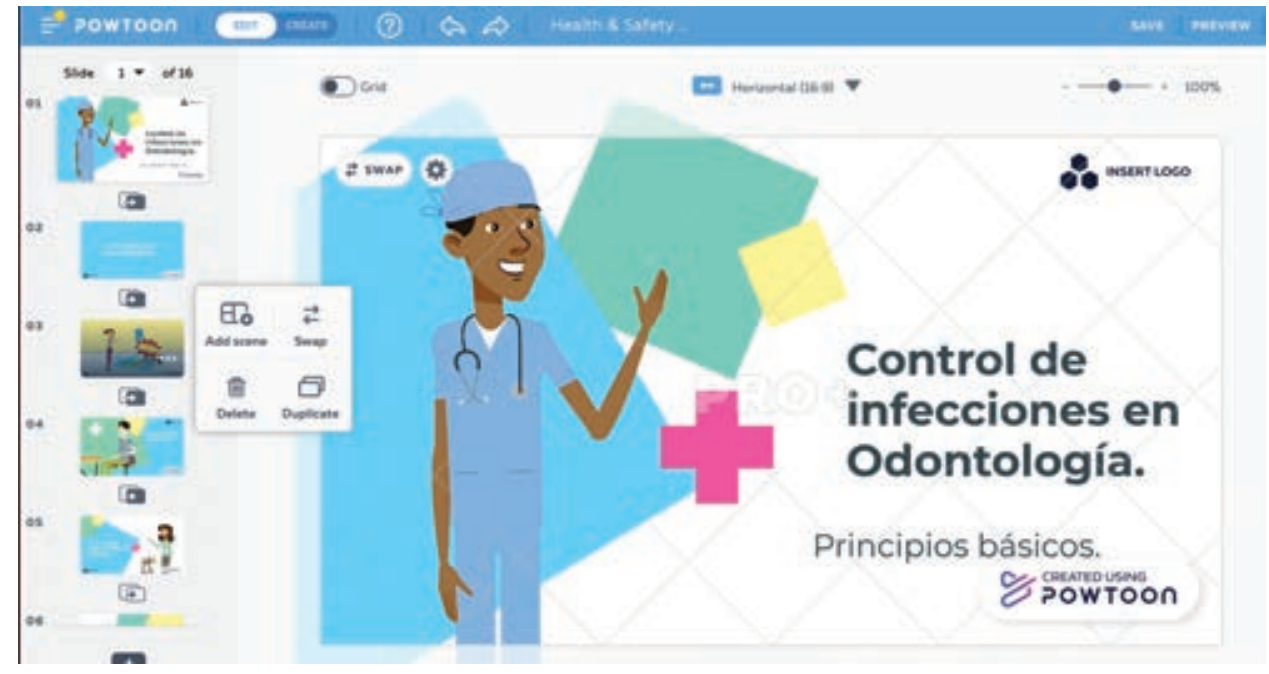

Figura 2:

Creación de sesiones interactivas a través de Powtoon.
En Internet existen diversas aplicaciones y plataformas para hacer más dinámicas las clases, rediseñando las presentaciones ya existentes o creando nuevas con una apariencia más dinámica y agradable que permite sin duda atraer la total atención por parte del estudiante, ejemplos de éstas son Nearpod, que permite la creación, mejoramiento de presentaciones, creación de «quizzes» de opción múltiple o preguntas abiertas, completar textos, dibujar, trabajo en equipo, entre otros. Otra plataforma muy dinámica y fuertemente recomendable es Powtoon, que permite al docente la creación de presentaciones interactivas dotadas de audio, movimiento y total dinamismo durante su reproducción (Figura 2).

Otra excelente opción para la realización de material interactivo por parte de los profesores o los alumnos es la creación digital de infografías a través de Piktochart (Figura 3). Una infografía es un elemento didáctico muy útil en pedagogía que permite combinar elementos visuales que aportan un despliegue gráfico de la información facilitando así el entendimiento de temas complejos. ${ }^{10}$

\section{Consideraciones en el uso de tecnologías educativas en la formación odontológica}

La actividad clínica de la carrera de odontología nunca podrá ser sustituida por ningún método educativo a distancia; no obstante, el aprendizaje teórico de nuestra profesión puede verse sumamente beneficiado mediante la implementación de la tecnología.

Además de la formación clínica, existen otras actividades de enseñanza habitualmente se realizan en la licenciatura en odontología como las sesiones teóricas impartidas por el profesor a cargo, exposiciones por parte de los alumnos, la discusión de casos clínicos, la revisión de la literatura científica actual en español e inglés y las prácticas en laboratorio.

Que el profesor cuente con videograbaciones y fotografías de resolución de casos clínicos en su práctica clínica a nivel público y/o privado para compartir con sus alumnos, puede ser un excelente método para despertar la curiosidad en los alumnos, además de fomentar su criterio clínico en la toma de decisiones. Este material audiovisual clínico puede ser proyectado y discutido durante sesiones virtuales en tiempo real o bien, mediante la creación de una técnica pedagógica conocida como Aprendizaje Basado en Problemas (ABP), el cual consiste en la presentación de un problema real que el alumno debe resolver mediante el análisis de caso y revisión de la literatura actual.

Asimismo, dependiendo del semestre o año que cursen los alumnos, pueden realizar videograbaciones y toma de fotografías ejecutando determinados procedimientos que les permitan desarrollar hasta cierto punto habilidad manual, por ejemplo, la apertura de cavidades y preparaciones de prótesis en tipodontos, el tallado de anatomía dental en cubos de cera o barras de jabón, el doblado de alambres para la fabricación de aparatos, entre otros.

Por otro lado, los profesores deben tener la suficiente madurez para comprender que no todos sus alumnos cuentan con las mismas posibilidades económicas y tecnológicas, y resulta importante exigir exclusivamente actividades que con seguridad todos los alumnos inscritos tendrán la posibilidad de entregar. Ésta es quizá una de 
las desventajas de la aplicación de tecnologías educativas conocida como brecha digital, donde las desigualdades socioeconómicas limitan a algunos alumnos a contar con todos los elementos necesarios para hacer uso de las TIC. ${ }^{11}$

\section{CONCLUSIÓN}

Como se habrá visto a lo largo del presente artículo, existen múltiples tecnologías educativas que pueden ser implementadas en la formación de los alumnos no so- lamente durante el periodo de pandemia, sino también posterior a ésta. Muchos de los odontólogos que han decidido dedicarse a la formación de nuevos profesionistas, carecen en su mayoría de conocimientos pedagógicos que favorezcan y faciliten el proceso de enseñanza-aprendizaje en el aula, por lo que la formación y actualización constante de los docentes por parte de la institución educativa donde laboran es de vital importancia.

Existe una infinidad de aplicaciones y plataformas que facilitan el proceso de enseñanza-aprendizaje en odontología y el primer paso para poder explotar sus beneficios

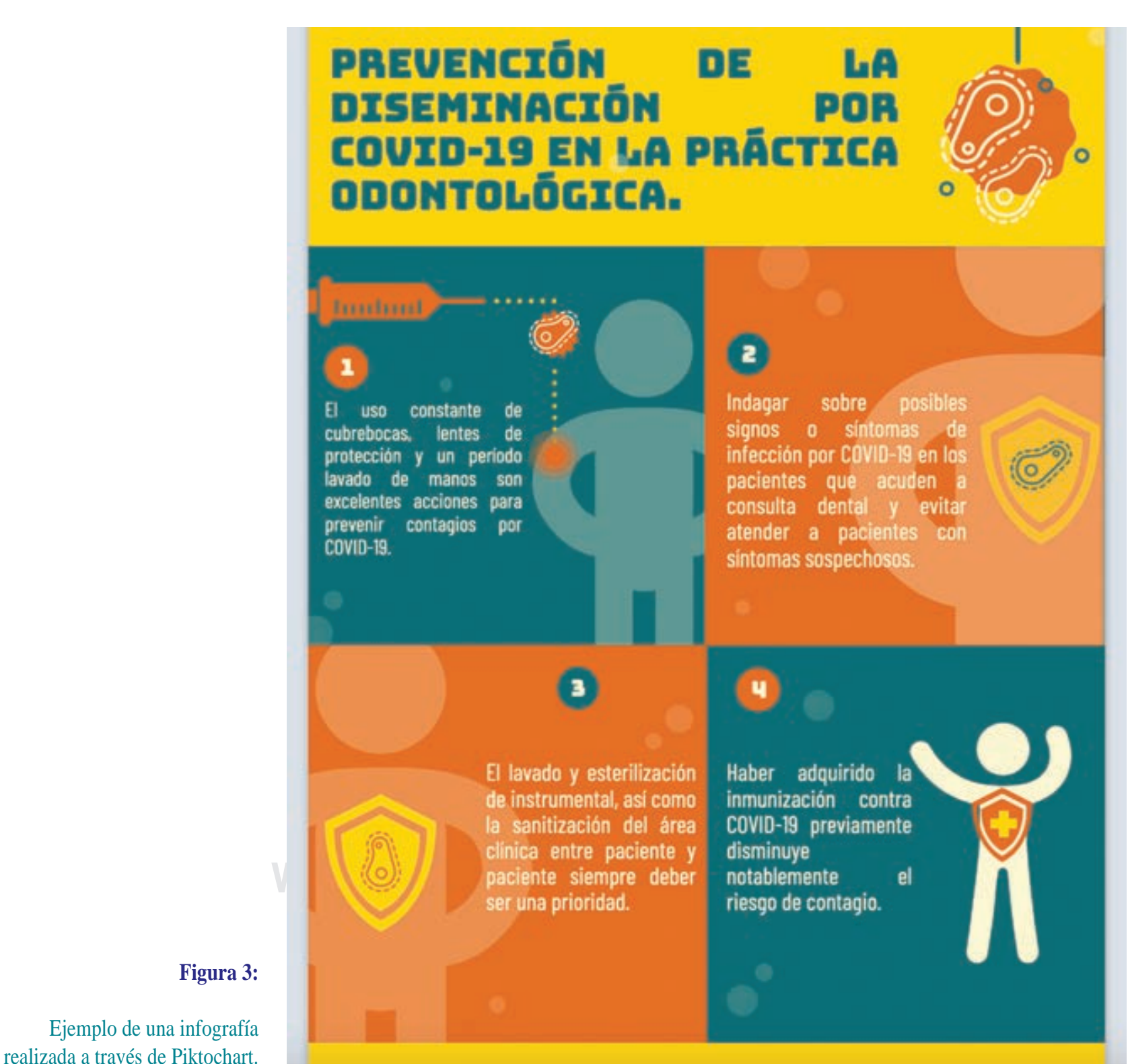


es sin duda que el docente tenga la mente abierta y la mejor disposición para aprender a utilizarlas.

\section{REFERENCIAS}

1. Cruz-López Y, Cruz-López AK. La educación superior en México. Tendencias y desafíos. Avaliacao. 2008; 13 (2): 293-311.

2. Spoletti BP. La enseñanza en la clínica odontológica. Rev E dc Cienc Salud. 2014; 11 (2): 166-170.

3. Vergara C, Zaror C. Proceso de enseñanza-aprendizaje en la clínica odontológica. Aspectos teóricos. Rev Educ Cienc Salud. 2008; 5 (1): 6-11.

4. Torres CPB, Cobo BJK. Tecnología educativa y su papel en el logro de los fines de la educación. Educere. 2017; 21 (68): 31-40.

5. Terrazas PR, Silva MR. La educación y la sociedad del conocimiento. Perspectivas. 2013; 32: 145-168.

6. Balladares-Burgos J. El aprendizaje híbrido y la educación digital del profesorado universitario. Revista Cátedra. 2018; 1 (1): 53-69.

7. Sánchez RJ. Plataformas de enseñanza virtual para entornos educativos. Pixel-Bit. Revista de Medios y Educación. 2009; 34: 217-233.
8. Ortíz-Colón AM, Jordán J, Agredal M. Gamificación en educación: una panorámica sobre el estado de la cuestión. Educ Pesqui Sao Paulo. 2018; 44: e173773.

9. Guzmán RJF. La necesidad de una reingeniería en el proceso de enseñanza aprendizaje. Realidad y Reflexión. 2010; 9 (30): 27-36.

10. Minervini MA. La infografía como recurso didáctico. Revista Latina de Comunicación Social. 2005; 8 (59): 59.

11. Toudert DE. Brecha digital y perfiles de uso de las TIC en México: un estudio exploratorio con microdatos. Culturales. 2015; 3 (1): 167-200.

Conflicto de intereses: El autor declara no tener ningún conflicto de intereses.

Aspectos éticos: Ninguno.

Financiamiento: Ninguno.

Correspondencia:

Agustín Tiol-Carrillo

E-mail: agustintiolcarrillo@gmail.com 